\title{
Evaluación del desempeño docente en la formación virtual: ideas para la configuración de un modelo
}

\section{Teacher performance evaluation in virtual training: setting a model}

\author{
Julio Cabero Almenara \\ $\mathrm{M}^{\mathrm{a}}$ del Carmen Llorente Cejudo \\ Juan Antonio Morales Lozano \\ Universidad de Sevilla, US (España)
}

\section{Resumen}

Conscientes de la significación de la actuación docente en los procesos formativos (pues es una pieza clave de su funcionamiento y éxito, además de ser un elemento esencial para cambiar, transformar y mejorar las prácticas educativas), y en los contextos de formación virtual en particular, nos adentramos en la temática de la evaluación del desempeño del docente que realiza su actividad profesional bien bajo la modalidad e-learning o b-learning. A partir de la revisión bibliográfica, nos aproximamos por una parte a la conceptualización y caracterización de los procesos docentes en la formación virtual y su evaluación, así como al análisis de diferentes estudios y propuestas que nos permitan identificar los elementos y estrategias a considerar en la construcción de un modelo evaluativo. Entendemos necesario plantear un modelo, que integrando las perspectivas de los diferentes agentes (profesor, estudiantes y directivos) y una combinación de instrumentos (cuestionarios, autoinformes, porta $<$ folios, resultados del aprendizaje), se estructure en torno a las siguientes dimensiones: Conocimiento disciplinar, Conocimiento Pedagógico, Conocimiento Tecnológico y Cumplimiento de Normas. Los análisis efectuados permitirán el establecimiento de políticas de evaluación del desempeño docente en procesos formativos bajo modalidad semipresencial u online; al mismo tiempo, se pueden establecer modelos de evaluación que sirvan como práctica pedagógica e investigadora, contemplando las visiones de sus diferentes actores: alumnos, docentes y directores o responsables académicos.

Palabras clave: enseñanza; e-learning; b-learning; personal docente; formación de profesores; Evaluación del profesor.

\section{Abstract}

This article discusses the evaluation of e-learning or b-learning teacher performance, given the significance of such performance both in the overall learning process (specifically in its development and success, and also in the change, transformation and improvement of educational practices), and in the various contexts of virtual education in particular. Based 
on a bibliography analysis, we approach the conceptualization and characterization of the educational processes in virtual training and its evaluation, and the studies and proposals that enable the identification of the elements and strategies to be considered in the construction of an evaluation model. We understand that it is necessary to propose a model that is structured around the following dimensions: disciplinary knowledge, pedagogical knowledge, technological knowledge and compliance performance, integrating the perspectives of different actors (teacher, students, authorities) and a combination of instruments (questionnaires, self-reports, portfolio, learning results). Analyses will allow the practice of policies on teacher performance evaluation under either blended learning processes or online. At the same time, it will be possible to adopt assessment models that serve for teaching and research practice, considering the views of the different actors: students, teachers, and academic authorities.

Keywords: teaching; e-learning; b-learning; teaching personnel; teacher education; teacher assessment.

\section{EVALUAR EL DESEMPEÑO DOCENTE}

Pretendemos adentrarnos en la temática de la evaluación del desempeño del docente universitario que realiza su actividad en un contexto de formación virtual (e-learning/b-learning), y aportar referencias conceptuales para la reflexión; conscientes de la transcendencia del docente para el funcionamiento, mejora y éxito de los procesos/prácticas educativas (Galán, González-Galán y Rodríguez, 2014; Tejedor, 2012; Vaillant y Marcelo, 2015), y de la escasez de propuestas (Marcimiack, 2016). Recientemente Murillo e Hidalgo (2015) han publicado un artículo, cuyo título es claramente significativo de la importancia que registra esta actividad académica: "Dime Cómo Evalúas y Te Diré Qué Sociedad Construyes", donde señalan: "La evaluación educativa es una actividad de marcado carácter político. Qué evalúes, cómo, cuándo, para qué, para quién... determina, y está determinada, por la sociedad que queremos, por nuestra utopía. Quien defiende la evaluación como una actividad meramente técnica cargada de palabras tales como validez, fiabilidad u objetividad, ya nos está diciendo desde qué posición ideológica parte y qué mundo desea" (Murillo e Hidalgo, 2015, p. 5).

Diversas han sido las definiciones sobre la evaluación; unas marcadas por el momento histórico en el cual se ofrecieron (pretyleriana, tyleriana, del realismo, objeto a evaluar,...), y otras por los conceptos implicados (objetivos perseguidos, alcance, actividades realizadas,...). Para Ruiz (2007) estas se pueden agrupar en cuatro grandes categorías: a) Centrada en el logro de los objetivos; b) Como emisión de juicios de valor o determinación de méritos; c) Como un proceso de recogida de información para la toma de decisiones; y d) Como aglutinación de algunas de las posiciones citadas anteriormente.

Cuando en el campo de la educación nos referimos a la evaluación, asumimos que con este término aludimos al “...proceso controlado y sistemático de análisis 
de la calidad de un servicio -educación- prestado a la sociedad que detecta sus atributos críticos inherentes, que los aprecia en base a criterios de valor y que orienta el esfuerzo indagador a estudiar las condiciones del servicio y a mejorar su funcionamiento" (Villar, 1994, 1-2). Siguiendo a Tejada (2005), entendemos la evaluación como una actividad procesual, que persigue la emisión de un juicio de valor para adoptar decisiones. No es exclusivamente una acción técnica, es una actividad humana y por tanto cargada de valores éticos, estéticos y axiológicos; sobre la que se debe reflexionar, como por ejemplo: criterios a movilizar, instrumentos a utilizar, realizadores de la evaluación, alcance y personas sobre las que se centrará.

En cuanto a los métodos utilizados (diversos), han girado en torno a tres grandes modelos: a) conductista-eficientista (consecución de objetivos de Tyler, de planificación educativa de Crombach, el CSE de Alkin,...); b) humanístico (Contraposición de Owens y Wolf, de atención al cliente de Scriven, artístico de Esiner,...); y c) holístico (de evaluación responderte de Stake, iluminativo de Parlett y Hamilton, y holístico de MacDonald,...). Debemos movernos en un modelo mixto, que persiga recoger información objetiva, tanto cuantitativa como cualitativa, de la satisfacción de los participantes, de la valoración de los diferentes componentes, y sobre la autopercepción del docente respecto a la calidad de su participación y ejecución.

Al mismo tiempo, para que la evaluación sea significativa debe cumplir una serie de características que, de acuerdo con Duart y Martínez (2001), podemos concretar en: a) Sistemática (no es un conjunto de acciones puntuales sino una actividad que debe hacerse periódicamente, en función de un programa previamente consensuado. Además, debe superar la dimensión descriptiva para cobrar sentido como herramienta al servicio del modelo pedagógico de la institución); b) Objetiva (se debe garantizar la objetividad de las evaluaciones, a lo que contribuirá la existencia de diversas fuentes de información); c) Participativa (toda la organización, empezando por el propio evaluado, debe participar en la definición e implantación del sistema); y d) Flexible (se trata de un sistema, no de una técnica, por lo que debe elegirse el método de evaluación en función de las características de la organización). En definitiva, hablar de evaluación no es referirnos meramente a aspectos técnicos, sino también reflexivos y organizativos.

La temática de la evaluación del desempeño docente ha evolucionado por diferentes fases y etapas: ha pasado de la evaluación de los docentes de primariasecundaria a los de universidad; de la utilización de modelos donde el único informante era el estudiante, a modelos donde se recoge información de diferentes fuentes/agentes; y de modelos donde solo se utilizaba un cuestionario a la aplicación de una variedad de ellos, para la combinación y triangulación de la información encontrada. Y ha evolucionado de una etapa en la que se trataba de identificar variables o factores asociados a la eficacia docente, a otra centrada en la transferencia de modelos de calidad de la empresa (EFQM), y a una perspectiva sistémica. Pero no 
debemos olvidarnos de diferentes contextuales que claramente influyen en la misma (Jornet, González-Such y Sánchez-Delgado, 2014).

Al hablar dela evaluación del docente universitario, nos encontramos inicialmente con una serie de problemas: la dificultad de conceptualizar qué podemos entender por buen profesor, los problemas inherentes a la medición de los conocimientos o aptitudes pedagógicas de un profesor, los problemas de carácter práctico que se nos presentan respecto a las estrategias más adecuadas que debemos seleccionar para llevar a cabo el proceso evaluador, y la existencia de la renombrada libertad de cátedra que para algunas personas es sinónimo de garantía de calidad y de la no necesidad de evaluación de la actividad que desempeñan. Reconociendo estas limitaciones y de acuerdo con Tejedor (2012, p. 319), podemos señalar que lo “...que sí tenemos muy claro respecto a la evaluación del profesorado es que se trata de un proceso que debe orientarse fundamentalmente a la estimación del nivel de calidad de la enseñanza a fin de contribuir progresivamente a su mejora”. Y “...no debe verse como una estrategia de vigilancia jerárquica que controla las actividades de los profesores, sino como una forma de fomentar y favorecer el perfeccionamiento del profesorado, como una manera de identificar las cualidades que conforman a un buen profesor para, a partir de ahí, generar políticas educativas que coadyuven a su generalización" (Tejedor, 2012, p. 321).

En definitiva, para hablar de evaluación del desempeño docente debemos tener en consideración diferentes aspectos que la pueden condicionar, como son el nivel en el que está desarrollando la acción formativa (grado, postgrado, master, doctorado); modalidad formativa (presencial, semipresencial o virtual); las funciones académicas de la institución (docencia, investigación, extensión y gestión); o que el profesor desempeñe su función a tiempo completo o parcial (Poblete, Carrasco, y Saelzer, 2007). Pero, en consonancia con lo expuesto anteriormente entendemos que la acción evaluadora tendrá una doble finalidad, por una parte, el mejoramiento de los aprendizajes que adquieren los estudiantes, y por otra, el perfeccionamiento del propio docente (Román y Murillo, 2008). La evaluación del desempeño es una herramienta fundamental para gestionar la calidad docente, cumpliendo al mismo tiempo una significación para la política de recursos humanos de la organización educativa (Duart y Martínez, 2001).

Como señalan Garrido y Fuentes (2008, p. 127): "se trata entonces de hacer de la evaluación un acto de aprendizaje. La evaluación del profesorado tiene como propósito el mejoramiento de su acción profesional. Se evalúa esencialmente para comprender y transformar las prácticas profesionales del docente, con el propósito formativo de mejorarlas y con ello la calidad de los aprendizajes de los estudiantes". 


\section{EL OBJETO DE LA EVALUACIÓN DEL DESEMPEÑO DOCENTE: PRESENTACIÓN DE ALGUNOS MODELOS DE EVALUACIÓN}

Si bien las dimensiones clásicas sobre las que recoger información han sido articuladas alrededor de tres grandes etapas: planificación, desarrollo y resultados; diferentes autores han realizado matizaciones al respecto, muchas de ellas derivadas dela diversidad defunciones que el profesor en la enseñanza virtual realiza: facilitador, diseñador, mentor, evaluador, motivador, moderador, orientador y experto (García y Pineda, 2011; Vaillant y Marcelo, 2015). Así Martí (2012), habla de cuatro grandes dimensiones: a) preparación de las clases; b) creación de un ambiente propicio para el aprendizaje de los alumnos; c) sistema de docencia que permita un aprendizaje de todos los alumnos a su cargo, y d) responsabilidades profesionales. Contreras (2010), al presentar el modelo de evaluación del desempeño de la Universidad Pontificia Católica de Valparaíso (Chile), contempla diferentes dimensiones: dominio disciplinario (conocimiento de la disciplina y su impacto en el aprendizaje de los estudiantes), planificación de la enseñanza (proceso que traza y dispone los elementos del currículum), enseñanza para el aprendizaje (conjunto de actividades planificadas para promover el aprendizaje), evaluación (análisis del proceso que el docente desarrolla para que sus estudiantes evidencien sus aprendizajes), ambiente de aprendizaje (el producto de las interacciones entre los integrantes de una clase, que generan emociones/sentimientos que impactan en los procesos de enseñanza/aprendizaje), y responsabilidad formal (se incorporan elementos como la puntualidad, la asistencia, o la entrega de pruebas/tareas de evaluación en plazos). Román y Murillo (2008) señalan los siguientes conocimientos básicos sobre los que se debe evaluar al docente: contenidos de su área de especialización; aspectos instrumentales considerados importantes para la docencia; características de los estudiantes a quienes va a enseñar; conocimientos sobre el proceso de enseñanza, y su formación respecto a las bases sociales de la educación. Por su parte Zúñiga y Jopia (2007), en una investigación donde preguntan a vicerrectores y decanos sobre los aspectos que creen que hay que evaluar, encuentran las siguientes coincidencias: el dominio de la disciplina, las competencias generales en docencia, el cumplimiento de objetivos, las metodologías utilizadas, la calidad de la enseñanza y la creación de atmósferas adecuadas para el aprendizaje.

Pero posiblemente sea el "Centro Interuniversitario de Desarrollo" (CINDA) el que ha sistematizado más las áreas respecto a las cuales se deben recoger información para una evaluación significativa del desempeño docente: 1) Planificación de las actividades docentes; 2) Ejecución de las Actividades Docentes; 3) Evaluación de los Aprendizajes (capacidad del profesor para asegurar que sus estudiantes logren aprendizajes significativos, dando fe pública de ello); 4) Evaluación de la Práctica Pedagógica; y 5) Compromiso con el proyecto educativo institucional (CINDA, 2007).

Por otra parte no podemos olvidarnos de la significación que están adquiriendo las capacidades y competencias como elementos caracterizadores de la cualificación 
docente, y al respecto Cano (2005), sintetizando las propuestas realizadas por diferentes autores, nos habla de las siguientes: 1) Capacidad de planificación y organización del propio trabajo; 2) Capacidad de comunicación; 3) Capacidad de trabajar en equipo; 4) Capacidad de establecer relaciones interpersonales satisfactorias y de resolver los conflictos; 5) Capacidad de utilizar las nuevas tecnologías de la información y la comunicación; 6) Autoconcepto positivo y ajustado; y 7) Autoevaluación constante de nuestras acciones. En esta línea, Marciniak (2015) apunta tres tipos de competencias que deben poseer los docentes que trabajan en contextos de formación virtual: 1) pedagógicas (profundizar/ investigar temas, estructurar el conocimiento, diseño de tareas individualizadas para el autoaprendizaje...), 2) técnicas (saber dirigir y participar en comunicaciones asincrónicas y sincrónicas, usar el software con propósitos determinados, diseñar y habilitar una clase virtual con recursos multimediales...), y 3) didácticas (redactar normas claras/precisas para evaluar la participación de los estudiantes en los foros y presentarlos a los estudiantes, elegir las formas de cooperación y comunicación con los estudiantes teniendo en cuenta los objetivos didácticos, respetar los períodos de tiempos establecidos en relación con el desarrollo del programa tanto relacionados con su propio trabajo, como con las actividades de los estudiantes...).

Específicamente referido a la educación a distancia y virtual, Mercado, Palmerín y Sesento (2011), nos hablan de la necesidad de un equilibrio entre la relación afectiva y cognoscitiva para el proceso efectivo de la tutoría, poseer experiencia docente y de investigación, con conocimiento del proceso de aprendizaje, y contar con habilidades para la comunicación ya que intervendrá en una relación humana y compleja mediada por tecnología.

Y en cuanto a los dominios que debe poseer este profesional para desenvolverse en entornos tecnológicos, en los últimos tiempos se está impulsando el denominado modelo TPACK ("Technological Pedagogical Content Knowledge) (Mishra y Koehler, 2006; Cabero y Barroso, 2016), que pone de manifiesto, por una parte, que los docentes deben poseer conocimientos a nivel disciplinar, de contenido, tecnológico y pedagógico; dimensiones que no se perciben de forma aislada sino en interacción y, por otra, que puede ser un buen modelo para la capacitación de los docentes en preservicio (Tokmak, Incikabi y Ozgelen, 2013; Zelkowski, Gleason, Cox, D.C. y Bismarck, 2013) (figura 1). 
Figura 1. Modelo TPACK de Mishra y Koehler (2006)

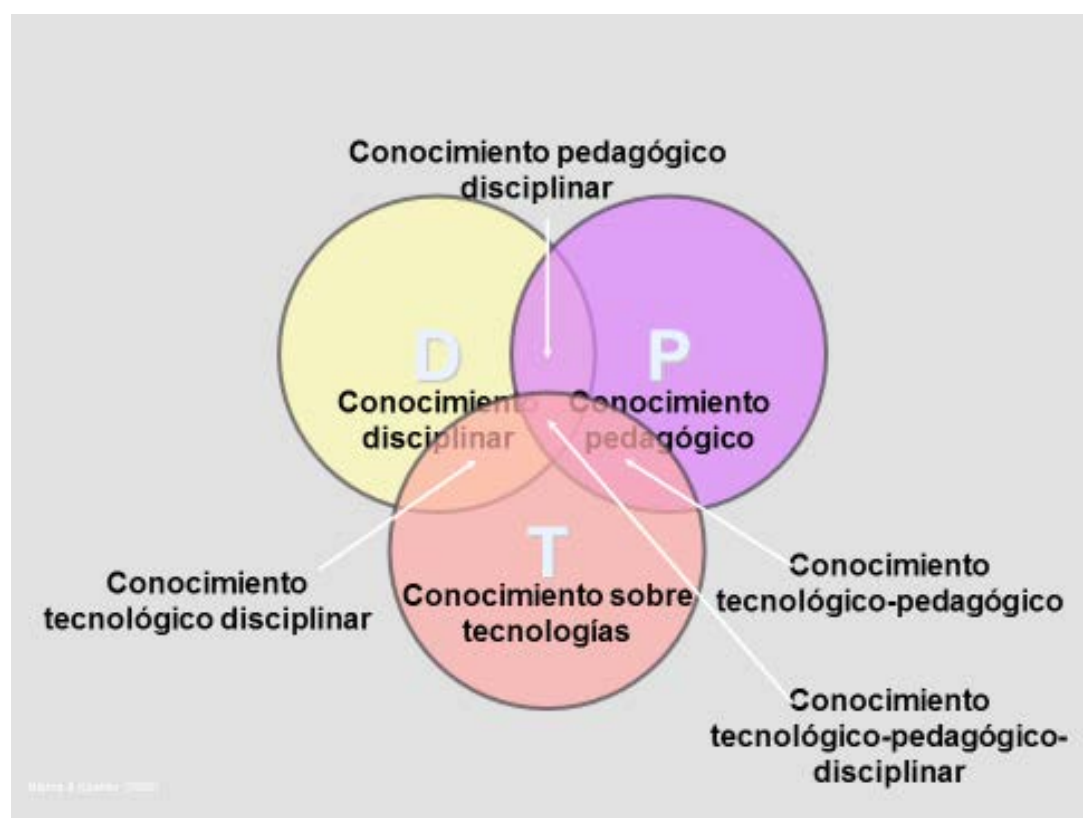

\section{EL PROCESO DE LA EVALUACIÓN DEL DESEMPEÑO DOCENTE}

Hemos de abordar dos aspectos relevantes, aunque estrechamente relacionados: los agentes (que han de proporcionar información) y los instrumentos (para recabarla). Por lo que se refiere al primero de los aspectos, si bien tradicionalmente los informantes fundamentales de la evaluación del desempeño de los docentes han sido los estudiantes a través de cuestionarios de satisfacción (Moreno-Murcia y otros, 2015; Tabera y otros, 2015); hemos de ser conscientes, por una parte, que no son las únicas opciones a considerar, y por otra, que los informantes deben ser variados, e incorporar la evaluación por pares, por responsables académicos (decanos, directores de departamentos), por exalumnos, por expertos, o la autoevaluación (CINDA, 2007; Toro, Backhouse y Valassina, 2007; Zúñiga y Jopia, 2007; Contreras, 2010; Montero, 2012; Tejedor, 2012; Caballero, 2013); y que los instrumentos a nuestro alcance son variados: cuestionarios, informes, entrevistas, portafolios... (Rueda, Luna, García y Loredo, 2010; Jarauta y Bozu, 2013; La Serna, Becerra, Beltrán y Zhang, 2014).

No podemos obviar, al margen de los cuestionamientos de su posible falta de objetividad o condicionalidad por algunas variables (Reyero, 2014), la relevancia de los estudiantes en los procesos de evaluación del desempeño docente, ya que son informantes claves en su calidad de beneficiarios directos de la acción formativa 
(CINDA, 2007; Tejedor, 2012; Moreno, Aracil y Reina, 2014); y constituyen una excelente fuente para percibir sus propios autoaprendizajes y percibir su opinión acerca de las buenas o malas prácticas pedagógicas. O como llaman la atención Muñoz y Jiménez (2012, p. 36), el estudiante “...no debe ser interpretado como un cliente pasivo frente a un producto de consumo, ya que en el caso de la docencia se establecen unas relaciones profesor-alumno y alumno-profesor. A esto hay que unir las relaciones de los alumnos entre sí, todo ello hace que el alumno deba ser considerado un "cliente proactivo". Y coincidimos con Gaytán cuando afirma que a pesar de las diferentes críticas y limitaciones, "las encuestas al alumnado pueden tener, si se usan de forma positiva (y no coercitiva, ni punitiva) un efecto beneficioso en la actividad del profesorado y de la calidad general del desempeño docente, si se emplean como un indicador más, del estado de la convivencia en el aula y el desarrollo del proceso de construcción del aprendizaje" (Gaytán, 2012, p. 51).

Son variados los referentes de la evaluación realizada por los estudiantes bajo la modalidad de cuestionarios de satisfacción. Así López-Barajas y Carrascosa (2005) elaboraron un cuestionario compuesto por 25 ítems desglosados en cuatro dimensiones: interacción con el alumnado, metodología, obligaciones docentes y evaluación, medios y recursos, para evaluar la docencia. En esta misma línea Casero (2008) diseñó un instrumento formado por 92 ítems agrupados en las siguientes dimensiones: infraestructura, programa, conocimiento/interrelación de la materia, metodología, materiales, actitud del profesor, evaluación, prácticas y satisfacción para evaluar a los docentes universitarios, buscando consenso entre estudiantes y docentes respecto a la importancia que les otorgaban. Recientemente MorenoMurcia (2015), ha llevado a cabo un estudio de evaluación de los docentes, donde construyeron un cuestionario conformado por 28 ítems que se agruparon en tres dimensiones: planificación, desarrollo, y resultado.

La evaluación del desempeño docente desde una perspectiva de mejora no puede obviar la información proporcionada por el propio docente evaluado, es decir, la autoevaluación a través de autoinformes o portafolios (Salazar, Hidalgo, Villalobos, Marín, Coloma, Mendivil, Pesci y Páez, 2014). Un proceso e instrumentos que, como señala Tejedor, posibilitan "a un profesor aportar información sobre su actividad docente. Suele estar pensado para conocer el modo que ha resuelto los problemas asociados a la planificación, el desarrollo y los resultados de su práctica. Al mismo tiempo, el docente puede aportar valoraciones y reflexiones importantes para la mejora de su práctica docente y para la organización de la docencia que se realiza en su propio centro...” (2012, p. 323). En definitiva, la autoevaluación es un proceso válido para la reflexión crítica del quehacer educativo, en el que los docentes formulan opiniones valorativas sobre la adecuación y efectividad de su propio conocimiento, actuación, principios o consecuencias con el fin de mejorarse a sí mismos, y por ende, nos facilita los elementos claves a contemplar a la hora de realizar las mejoras en el desempeño del docente. Entre las razones que pueden justificar la conveniencia de evaluarse a sí mismo, podríamos destacar: 
a. Realizar un proceso de toma de conciencia de lo que se está haciendo, así como de los objetivos que se pretenden alcanzar.

b. Proceso de reflexión que le permite al docente establecer modificaciones/ cambios en las planificaciones.

c. Constituir adaptaciones sobre los proyectos institucionales a las condiciones de la práctica docente.

d. Reflexionar sobre sus fortalezas/debilidades.

e. Percibir de manera fiable y con honestidad su actuación en el aula con el resto de alumnos.

f. Reflexionar sobre sus necesidades profesionales y buscar/crear estrategias para satisfacerlas.

Y entre los riesgos/limitaciones debemos señalar la autocomplacencia apuntada por CINDA (2007) o su falta de objetividad (los individuos tienden a considerarse buenos profesionales); y como apunta Barber (1997): la evaluación puede convertirse en una forma de auto-justificación, los docentes mediocres tienden a ser menos exactos en su autoevaluación que los profesores competentes, si se utiliza un asesor hay tendencia a depender de este en lo que a la realización de la evaluación se refiere, posee un potencial inherente de autoinculpación si los datos se utilizan sumativamente, dificultad para cuantificar la evaluación y tendencia a centrar la evaluación en los aspectos externos (presencia personal, maneras, etc.).

Como exponen Airasian y Gullickson (1999, p. 15), la autoevaluación es una de las formas más comunes de evaluación del profesorado. Ésta posee su eje principal en la auto-comprensión y mejora de uno mismo como actividades de importancia crucial. Así pues, este proceso comprende y queda estructurado a través de diferentes fases:

a. Identificación del problema/delimitación: etapa en la que surge una sensación de incomodidad, curiosidad o un deseo de cambio. El profesor debe enfocar el tema que se va a tratar, identificar la información adecuada a recoger, y delimitar los criterios que pueden ayudar a identificar el éxito o el profesor hacia el objetivo deseado. La pregunta clave es: ¿Qué se va a evaluar?

b. Recogida/obtención de información: los datos o la información necesaria para informar el área de la práctica objeto de estudio se recogen y organizan. Los datos recogidos pueden ser formales o informales, pero se pone el énfasis en la obtención de evidencias de tipo formal. La pregunta clave es: ¿Qué información se va a recoger?

c. Reflexión/toma de decisiones: el profesor debe tomar una decisión referida a la práctica, principios, o consecuencias a examinar. La pregunta clave es: ¿Qué significado tiene esta información para mi práctica docente?

d. Aplicación/cambio: se hacen planes para llevar a cabo los cambios necesarios en la práctica que se derivan de las etapas anteriores. La pregunta clave es: ¿Qué acción, si cabe, se necesita ahora? 
Para llevar a cabo la autoevaluación existe una diversidad de propuestas de acuerdo a los instrumentos y técnicas a emplear (Solabarrieta, 1996; Barber, 1997; Díaz Alcaraz, 2007). En este sentido Solabarrieta (1996) nos habla de cuatro modelos: a) Basados en los juicios realizados por los propios profesores a partir de cuestionarios de autoevaluación; b) Utilización de grabaciones de audio y video de la propia actuación; c) Feedback externo procedente de observaciones o valoraciones, predominantemente cuantitativas, de compañeros, supervisores o alumnos; y d) Modelos definidos en términos generales como cualitativos. En el siguiente cuadro se presenta una síntesis de modelos y estrategias de autoevaluación efectuada por el mismo autor:

Cuadro 1. Clasificación de los modelos y estrategias de autoevaluación

\begin{tabular}{|c|c|c|}
\hline $\begin{array}{l}\text { ESTRATEGÍAS } \\
\text { BÁSICAS }\end{array}$ & $\begin{array}{l}\text { TÉCNICAS } \\
\text { POSIBLES }\end{array}$ & $\begin{array}{l}\text { DESCRIPCIÓN DE LAS } \\
\text { TÉCNICAS }\end{array}$ \\
\hline \multirow{3}{*}{$\begin{array}{l}\text { EVALUACIÓN } \\
\text { INDIVIDUAL }\end{array}$} & Reflexión personal & $\begin{array}{l}\text { El profesor considera abierta y } \\
\text { honestamente su actuación y genera } \\
\text { ideas. }\end{array}$ \\
\hline & $\begin{array}{l}\text { Análisis de grabaciones } \\
\text { de clases }\end{array}$ & $\begin{array}{l}\text { El profesor graba, observa e } \\
\text { interpreta interacciones de clases } \\
\text { orales y no orales. }\end{array}$ \\
\hline & $\begin{array}{l}\text { Lista de comprobación } \\
\text { para la autoevaluación }\end{array}$ & $\begin{array}{l}\text { El profesor describe y puntúa varios } \\
\text { actos de enseñanza y aprendizaje. }\end{array}$ \\
\hline \multirow{2}{*}{$\begin{array}{l}\text { EVALUACIÓN DE } \\
\text { RETROALIMENTACIÓN } \\
\text { (FEEDBACK) }\end{array}$} & Alumno & $\begin{array}{l}\text { El profesor obtiene información } \\
\text { específica de los alumnos sobre el } \\
\text { proceso de enseñanza a través de } \\
\text { diálogos, grabaciones, encuestas, } \\
\text { etc. }\end{array}$ \\
\hline & $\begin{array}{l}\text { Profesor, colegas, } \\
\text { Supervisor, Director }\end{array}$ & $\begin{array}{l}\text { El profesor obtiene información } \\
\text { sobre conceptos generales y } \\
\text { específicos mediante discusiones, } \\
\text { grabaciones, encuestas, } \\
\text { observaciones, intercambios, etc. }\end{array}$ \\
\hline \multirow{2}{*}{$\begin{array}{l}\text { EVALUACIÓN } \\
\text { INTERACTIVA }\end{array}$} & Supervisión & $\begin{array}{l}\text { El profesor solicita la presencia } \\
\text { del Supervisor para compartir y } \\
\text { analizar cooperativamente una } \\
\text { actividad. }\end{array}$ \\
\hline & Microenseñanza & $\begin{array}{l}\text { El profesor analiza una lección } \\
\text { simplificada con los alumnos, } \\
\text { colegas y / o supervisor para } \\
\text { mejorar una actividad futura. }\end{array}$ \\
\hline
\end{tabular}

Fuente: Adaptado de Solobarrieta (1996). 
En línea con una autoevaluación del desempeño docente desde una perspectiva de mejora, hemos de considerar el portafolio como un instrumento adecuado y pertinente (Cano, 2005; Villar y Alegre, 2012; Cabero y Marín, 2015; Pérez Gómez, 2016) para la mejora del desarrollo profesional del docente en la formación virtual ya que facilitan que los profesores reflexionen sobre su práctica (Bozu e Imbernon, 2012; Jarauta y Bozu, 2013).

Entendemos el portafolio, no solamente como una simple presentación y catalogación de trabajos del profesor (agrupamiento de materiales), aunque sean de diferentes tipologías (documentos, clips de vídeo, presentaciones, etc.) (Cano, 2005; Villar y Alegre, 2012; Pérez Gómez, 2016), sino además que dichos materiales deben expresar la situación «real» del grado de conocimiento de la persona o de las producciones realizadas a lo largo de un periodo de tiempo, de sus éxitos y fracasos, de sus posibilidades y limitaciones; es decir, que deben ser significativos, positivos y negativos, para explicar y comprender la evolución de una persona en la realización de una actividad o en su forma de participación en un proceso. Al mismo tiempo, los materiales deben estar cronológicamente ordenados para permitir a la persona que los observe y analice hacerse una idea de la evolución temporal de la actividad efectuada por el docente. En definitiva, no solo se trata de una recopilación de materiales sino de un instrumento para promover las valoraciones reflexivas respecto a la calidad y pertinencia de las actividades y materiales realizados (Pujolà y González, 2006).

Así mismo, no podemos olvidarnos de la eficacia que una gran mayoría de autores (Cebrián, 2011; Meeusa, Petegema y Engelsb, 2009; Martínez y Raposo, 2010) conceden al portafolio para la evaluación de los aprendizajes por competencias, ya que se ha percibido como una herramienta significativa para el análisis de su propio proceso de aprendizaje y la presentación de evidencias para la exposición de las competencias y capacidades adquiridas.

El portafolio puede cumplir tanto una función sumativa (certificación, selección, promoción, valoración...) como formativa, así como rendición de cuentas de lo que se ha hecho para reforzar los procesos de enseñanza y aprendizaje (Klenowski, 2005).

En la actualidad, y gracias al avance que han tenido las tecnologías, por un lado, por el aumento de su presencia en los contextos educativos y, por otro, por su amigabilidad y facilidad de uso, los portafolios electrónicos, e-portafolios, se están convirtiendo en la forma de evaluación por excelencia de los procesos de aprendizaje; puesto que nos permiten incorporar una diversidad de medios y recursos, disponer de amplitud de espacio, y de facilidad de traslado y actualización (Chang, Tseng, Liang y Liao, 2013; Jenson, 2011).

Por su parte, Barberá y Martín (2009) diferencian los e-portafolios en función de su finalidad (portafolio de producto y de proceso), según el emisor (personal y colectivo), según su audiencia (confidencial y público), según su contenido (de especialidad profesional, disciplinas académicas y temas específicos), según el 
soporte tecnológico en el cual son presentados (de software, de archivos en soporte CD-DVD, o aplicación virtual) y formatos (texto, imágenes, vídeos, MP3 y WAV).

En definitiva, apostamos por un portafolio del docente de formación virtual con una finalidad formativa/evaluativa, académico/profesional, selectivo, digital y semiestructurado; con base en las dimensiones del modelo: Conocimiento disciplinar del contenido, Conocimiento pedagógico, Conocimiento tecnológico y Cumplimentación de reglamentación y normas.

\section{UNAS REFLEXIONES FINALES PARA LA CONFIGURACIÓN DE UN MODELO DE DESEMPEÑO DOCENTE PARA LA FORMACIÓN VIRTUAL}

Hablar de formación virtual, como nos indica García Aretio (2014), es hablar de un sistema tecnológico de comunicación masiva y bidireccional, que sustituye la interacción personal en el aula como medio preferente de enseñanza, por la acción sistemática y conjunta de diversos recursos didácticos y el apoyo de una organización tutorial, que propician el aprendizaje autónomo de los estudiantes. Y en este contexto, como señalan Duart y Martínez (2001): “...la necesidad de evaluar la calidad docente se incrementa, si cabe, aún más. La universidad virtual se articula como una organización en red, en la que la docencia está a cargo de numerosos profesionales que, en el marco del modelo pedagógico de la institución, realizan su actividad docente a tiempo parcial". La evaluación en este contexto requiere que no olvidemos el componente tecnológico, que nos va a llevar a analizar una serie de aspectos, como: qué tipo de interacción establece y favorece el docente con sus estudiantes, qué materiales son los que los docentes les presentan a sus alumnos, etc. Para ello contamos con la posibilidad de analizar las acciones que los docentes realizan en los LMS: interacciones, número de participaciones, elementos tecnológicos que utiliza de la plataforma, herramientas de comunicación de la plataforma movilizada, etc. (Landeta, Palazio y Cabero, 2013). Interacción que, como ha sugerido García Aretio (2014), es clave para el desarrollo de la acción formativa.

Refiriéndonos a la evaluación del docente en la formación virtual, lo primero a señalar es que son más bien limitadas (Marciniak, 2016). Además, como señala Paéz (2010): "No es lo mismo el trato interpersonal con presencia física, que con ausencia y mediatización. Lo presencial, si es funcional a la personalización, corrige sobre la marcha el accionar docente y la reacción discente por evidencias del entorno y de las contingencias. La virtualidad, aunque sea por videoconferencia, ejerce "distancia" entre los sujetos; una pantalla, un monitor, puede aproximar lo que ocurre al "otro lado", pero es un clima artificial que aproxima datos por tecnofactos más o menos actualizados. Error en trasladar los principios y las estrategias empleados en la modalidad presencial a la forma virtual. Se requiere de una concepción diferente, aunque siempre educativa del sistema instructivo, que debe apuntar a la optimización de todas las funciones psicológicas superiores" (Paéz, 2010, p. 153). 
Realizados estos comentarios, y a manera de síntesis, señalar que somos partidarios de un modelo de evaluación del desempeño docente en el que:

a. Se recoja información de diferentes actores, fundamentalmente del profesor, de los estudiantes y de los responsables de las facultades y departamentos; es decir, de un modelo de evaluación que contemple lo externo (evaluación que recoja información del grado de satisfacción mostrado por los estudiantes en la acción formativa), interna (evaluación del desempeño docente por parte de los directores de centros, decanos, responsables de la formación virtual,...), y autoevaluación por parte del docente que ha realizado la acción formativa.

b. Se utilicen diferentes tipos de instrumentos como son: cuestionarios de satisfacción docente por parte de los alumnos, autoinformes de los docentes, portafolios donde se incorporen diferentes tipos de actividades realizadas por los profesores, y documentos de evaluación por parte de los decanos, directores de centros y responsables de la formación virtual.

c. Contemple los resultados académicos alcanzados por los estudiantes. Aunque, en este caso, sabiendo que es un indicador de la eficacia de la acción docente, debe ser tratado con cuidado.

d. Y que asuma la existencia de diferentes roles en los docentes: tutor, asesor, moderador en línea, experto en contenidos, evaluador, o director de la acción formativa.

La evaluación del desempeño del docente, que realiza su actividad profesional bajo la modalidad de la formación virtual (e-learning/b-learning), debe permitir recoger las diferentes miradas sobre la actuación del docente; en concreto: la visión del alumno, la autoevaluación del docente y la evaluación de directores o responsables académicos, donde el docente realiza su actividad profesional. Y para ello, hemos de recabar información de una serie de dimensiones (ver cuadro 2) para lo que hemos de servirnos de los siguientes instrumentos:

- Cuestionario de satisfacción del estudiante.

- Cuestionario de evaluación del responsable académico.

- Portafolio de actividades realizadas por el docente. 
Cuadro 2. Dimensiones de la evaluación del desempeño del docente virtual

\begin{tabular}{|l|l|l|}
\hline \multicolumn{1}{|c|}{ Dimensiones } & \multicolumn{1}{c|}{ Subdimensiones } \\
\hline Conocimiento disciplinar & - & \multicolumn{1}{c|}{ Dominio del contenido/Conocimiento de la materia. } \\
del contenido & trabajo.
\end{tabular}

Por lo que se refiere a su aplicación, debe efectuarse en diferentes momentos del acto educativo, de manera que pueda servirnos tanto para las evaluaciones de acciones específicas, el perfeccionamiento del profesorado, como para la promoción interna de los docentes. Ello exigirá la elaboración de propuestas evaluativas que sean fáciles y rápidas de aplicar, y de ofrecer los resultados a los docentes, para su mejora educativa.

Para finalizar, señalar, como apuntan diferentes autores (CINDA, 2007; Tejedor, 2012) un modelo de evaluación del desempeño docente como el que proponemos, presenta una serie de ventajas:

- Justo, ya que la información obtenida procede de diversas fuentes, lo que ayuda a eliminar sesgos.

- Fiable y consistente, al proceder del conjunto de agentes en contacto con la persona evaluada, los resultados de la evaluación proporcionan una información más precisa sobre la calidad real de la docencia. 
- Creíble, puesto que resulta más difícil rechazar una sugerencia de mejora si ésta viene avalada por las opiniones y sugerencias de diferentes personas y también por el propio implicado.

- Motivador, al facilitar un clima de participación y colaboración en el que todos pueden aportar mejoras constructivas.

- Valioso, ya que persigue la mejora de la enseñanza y, por tanto, aumentar la satisfacción y la calidad de los aprendizajes en los estudiantes.

\section{REFERENCIAS BIBLIOGRÁFICAS}

Airasian, P. W., y Gullickson, A. R. (1999). Herramientas de autoevaluación del profesorado. Bilbao: Mensajero.

Barber, L. W. (1997). Autoevaluación. En J. Millman y L. Darling-Hammond, Manual para la evaluación del profesorado (300315). Madrid: La Muralla.

Barberá, E., y Martín, E. de (2009). Portfolio electrónico: aprender a evaluar el aprendizaje. Barcelona: UOC.

Bozu, Z., e Imbernón, F. (2012). El portafolio docente como estrategia formativa innovadora del profesorado novel universitario. Un estudio de casos. Revista de Educación, 358, 238-257.

Caballero, K. (2013). Nivel de satisfacción del profesorado universitario hacia los sistemas de evaluación. Revista de Educación, 360, 483-508.

Cabero, J., y Barroso, J. (2016). ICT teacher training: a view of the TPACK model / Formación del profesorado en TIC: una visión del modelo TPACK. Cultura y Educación / Culture and Education, 28(3), 633-663.

Cabero, J., y Marín, V. (2015). E-Portfolios. Madrid: Ediciones CEF.

Cano, E.(2005).Elportafolio del profesorado universitario. Un instrumento para la evaluación y para el desarrollo profesional. Barcelona: Octaedro.

Casero, A. (2008). Propuesta de un cuestionario de evaluación de la calidad docente universitaria consensuado entre alumnos y profesores. Revista de Investigación Educativa, 26, 25-44.
Cebrián, M. (2011). Supervisión con e-portafolios y su impacto en las reflexiones de los estudiantes en el Practicum. Estudio de caso. Revista de Educación, 354, 183-208.

Chang, Ch., Tseng, K., Liang, Ch. y Liao, Y. (2013). Constructing and evaluating online goal-setting mechanisms in webbased portfolio assessment system for facilitating self-regulated learning. Computers \& Education, 69, 237-249.

CINDA (2007). Evaluación del desempeño docente y calidad de la docencia universitaria. Santiago de Chile: CINDA.

Contreras, G. (2010). Diseño y operación de un sistema de evaluación del desempeño docente con fines formativos: la experiencia de la Pontificia Universidad Católica de Valparaíso, Chile. Revista Iberoamericana de Evaluación Educativa, 3(1), 179-191.

Díaz Alcaraz, F. (2007). Modelo para autoevaluar la práctica docente. Madrid: Praxis.

Duart, J., y Martínez, M. J. (2001). Evaluación de la calidad docente en entornos virtuales de aprendizaje. Recuperado de http://www.uoc.edu/web/esp/art/ uoc/0109041/duartmartin.html

Galán,A., González-Galán, M.A.,y Rodríguez, P. (2014). La evaluación del profesorado universitario en España. Sistema nacional y divergencias territoriales. Revista de Educación, 366, 136-164. 
García Aretio, L. (2014). Bases, mediaciones y futuro de la educación a distancia en la sociedad digital. Madrid: Síntesis.

García, B., y Pineda, V. (2011). Evaluación de la docencia en línea: retos y complejidades. Revista Iberoamericana de Educación a Distancia, 14(2), 63-76.

Garrido, O., y Fuentes, P. (2008). La evaluación docente. Un aporte a la reconstrucción de prácticas pedagógicas más efectivas. Revista Iberoamericana de Evaluación Educativa, 1(2), 125-136.

Gaytán, S. (2012). Evaluar la docencia: estudio meta-evaluador del desarrollo del proceso de encuestas al alumnado y su fiabilidad en el Grado en Biología de la Universidad de Sevilla. En A. Castro y otros (Coords.), Calidad, evaluación y encuestas de la docencia universitaria (41-53). Murcia: Laborum.

Jarauta, B., y Bozu, Z. (2013). Portafolio docente y formación pedagógica inicial del profesorado universitario. Un estudio cualitativo en la Universidad de Barcelona. Educación XX1, 16(2), 343-362.

Jenson, J. D. (2011). Promoting selfregulation and critical reflection through writing students' use of electronic portfolio. International Journal of ePortfolio, 1(1), 49-6o.

Jornet, M., González-Such, J., y SánchezDelgado, P. (2014). Factores contextuales que influyen en el desempeño Docente. Revista Iberoamericana de Evaluación Educativa, 7(2), 185-195.

Klenowski, V. (2005). Desarrollo de portafolios para el aprendizaje y la evaluación. Madrid: Narcea.

La Serna, K., Becerra, A., Beltrán, A., y Zhang, H. (2014). La relación de las encuestas de evaluación docente con el rendimiento académico: La evidencia empírica en la Universidad del Pacífico. Revista Iberoamericana de Evaluación Educativa, 7(2), 105-115.
Landeta, A., Palazio, G., y Cabero, J. (2013). Plataformas tecnológicas. Madrid: Ediciones CEF.

López-Barajas, D., y Carrascosa, J. (2005). La evaluación de la docencia universitaria. Dimensiones y variables más relevantes. Revista de Investigación Educativa, 23(1), 57-84.

Marciniak, R. (2015). La educación superior virtual en Polonia: Condiciones de su organización, funcionamiento y evaluación. Memorias del Encuentro Internacional de Educación a Distancia, 4, 1-13.

Marciniak, R. (2016). Autoevaluación de programas de educación universitaria virtual. [tesis doctoral no publicada]. Barcelona: Universidad de Barcelona.

Martí, J (2012) Criterios para evaluar profesionalmente a los docentes. Recuperado de http://www.xarxatic.com criterios-para-evaluar-profesionalmentea-un-docente

Martínez, E., y Raposo, M. (2010). La rúbrica como recurso en la tutoría: percepciones del alumnado. Seminario internacional Las rúbricas de evaluación en el desempeño de competencias. Ámbitos de investigación y docencia (401-406). Bilbao: Universidad del País Vasco.

Meeusa, W. P., Peter Van y Engels, N. (2009). Validity and reliability of portfolio assessment in pre-service teacher education. Assessment \& Evaluation in Higher Education, 4(34), 401-413.

Mercado, H., Palmerín, M., y Sesento, L. (2011). La tutoría grupal en la educación. Cuadernos de Educación y Desarrollo, 3(31). Recuperado de http://www.eumed. net/rev/ced/31/vcg.html

Mishra, P., y Koehler, M. J. (2006). Technological Pedagogical Content Knowledge: A Framework for Teacher Knowledge. Teachers College Record, 108(6), 1017-1054.

Montero, A. M. (2012). El sistema de evaluación de la docencia y del 
profesorado: planificación y realización. En A. Castro y otros (Coords.), Calidad, evaluación y encuestas de la docencia universitaria (101-112). Murcia: Ediciones Laborum.

Moreno Murcia, J. A., Aracil, A., y Reina, R. (2014). La cesión de responsabilidad en la evaluación: una estrategia adaptada al Espacio Europeo de Educación Superior. Educación $X X 1,17(1), 183-200$.

Moreno-Murcia, J. A., Silveira, Y., y Belando, N. (2015). Cuestionario de evaluación de las competencias docentes en el ámbito universitario. Evaluación de las competencias docentes en la universidad. New approaches in educational research, 4(1), 60-66.

Murillo, F. L., e Hidalgo, N. (2015). Dime Cómo Evalúas y Te Diré Qué Sociedad Construyes. Revista Iberoamericana de Evaluación Educativa, 8(1), 5-9.

Páez, R.O.(2010). Evaluación delas funciones docentes en entornos instructivos virtuales (eiv). Revista Iberoamericana de Evaluación Educativa, 3(1), 147-158.

Pérez Gómez, A. (dir.) (2016). El portafolios educativo en Educación Superior. Madrid: Akal.

Poblete, A., Carrasco, S., y Saelzer, R. (2007). La problemática del desempeño docente: desafíos frente al requerimiento de la evaluación. En CINDA, Evaluación del desempeño docente y calidad de la docencia universitaria (30-32). Santiago de Chile: CINDA.

Reyero, D. (2014). La excelencia docente universitaria. Análisis y propuestas para una mejor evaluación del profesorado universitario. Educación XX1, 17(2), 125143.

Román, M., y Murillo, F. J. (2008). La evaluación del desempeño docente: objeto de disputa y fuente de oportunidades en el campoeducativo. RevistaIberoamericana de Evaluación Educativa, 1(2), 1-6.

Rueda, M., Luna, E., García, B., y Loredo, J. (2010). La evaluación de la docencia en las universidades públicas mexicanas: un diagnóstico para su comprensión y mejora. Revista Iberoamericana de Evaluación Educativa, 3(1), 77-92.

Ruiz, C. (2007). Evaluación de la formación. En J. Tejada y V. Giménez (Coords.), Formación de formadores. Escenario de Aula (649-701). Madrid: Thompson.

Salazar, J., Salazar, P., Hidalgo, C., Villalobos, A., Marín, P., Coloma, C., Mendivil, L., Pesci, G., y Páez, R. (2014). Reflexión pedagógica y autoevaluación docente: ¿Simbiosis o depredación? Revista Iberoamericana de Evaluación Educativa, 7(2), 147-155.

Solobarrieta, J. (1996). Modelos de evaluación del profesor. En F. J. Tejedor y J. L. Rodríguez Diéguez (Eds.), Evaluación Educativa II. Evaluación Institucional. Fundamentos teóricos y aplicaciones prácticas (173-182). Salamanca: IUCEUniversidad de Salamanca.

Tabera, M. V., y otros (2015). Percepciones de los estudiantes universitarios de Ciencias de la Salud sobre las actitudes de los docentes y su influencia en el clima de aprendizaje. Revista Complutense de Educación, 26(2), 275-293.

Tejada, J. (2005). Didáctica-curriculum. Diseño, desarrollo y evaluación curricular. Barcelona: Da Vinci.

Tejedor, F. (2012). Evaluación del desempeño docente. Revista Iberoamericana de Evaluación Educativa, 5(1), 319-327.

Tokmak, H., Incikabi, L., y Ozgelen, S. (2013). An investigation of change in mathematics, science, and literacy education pre-service teachers' TPACK. Asian-Pacific Education Researcher, 22(4), 407-415.

Toro, C., Backhouse, P., y Valassina, F. (2007). Recomendaciones para la implementación de un modelo de evaluación del desempeño docente. En CINDA, Evaluación del desempeño docente y calidad de la docencia 
universitaria (178-182). Santiago de Chile: CINDA.

Vaillant, D., y Marcelo, C. (2015). El ABC y D de la formación docente. Madrid: Narcea.

Villar, L. M. (1994). Naturaleza, modelos y fuentes para captar la evidencia de la evaluación educativa. En L. M. Villar (Dir.), Manual de entrenamiento: evaluación de procesos y actividades educativas (1-37). Barcelona: PPU.

Villar, L. M., y Alegre, O. M. (2012). Los portafolios electrónicos en el hemisferio de la evaluación auténtica. Madrid: Síntesis.
Zelkowski, J., Gleason, J., Cox, D. C., y Bismarck, S. (2013). Developing and validating a reliable TPACK instrument for secondary mathematics preservice teachers. Journal of Research on Technology in Education, 46(2), 173-206. Zúñiga, M., y Jopia, B. (2007). La evaluación del desempeño docente en las universidades chilenas: diagnostico desde la perspectiva de las autoridades universitarias. En CINDA, Evaluación del desempeño docente y calidad de la docencia universitaria (33-56). Santiago de Chile: CINDA.

\section{PERFIL ACADÉMICO Y PROFESIONAL DE LOS AUTORES}

Julio Cabero Almenara. Doctor en Ciencias de la Educación. Catedrático de Didáctica y Organización Educativa en la Universidad de Sevilla. Director del Secretariado de Recursos Audiovisuales y Nuevas Tecnologías de la Universidad de Sevilla. Director del Grupo de Investigación Didáctica: Análisis Tecnológico y Cualitativo de los Procesos de Enseñanza-Aprendizaje (Plan Andaluz de Investigación: HUM-390). Director de la Revista Pixel-Bit. Revista de Medios y Educación. Más información del CV en: http://investigacion.us.es/sisius/sis showpub.php?idpers $=1943$

E-mail: cabero@us.es

$M^{\mathbf{a}}$ del Carmen Llorente Cejudo. Doctora en Ciencias de la Educación. Profesora Titular del Departamento de Didáctica y Organización Educativa en la Universidad de Sevilla. Miembro del Grupo de Investigación Didáctica: Análisis Tecnológico y Cualitativo de los Procesos de Enseñanza-Aprendizaje (Plan Andaluz de Investigación: HUM-390). Miembro del Consejo Científico de la Revista PixelBit. Revista de Medios y Educación. Más información del CV en http://investigacion. us.es/sisius/sis showpub.php?idpers=10866

E-mail: karen@us.es

Juan A. Morales-Lozano. Doctor en Ciencias de la Educación. Profesor Titular del Departamento de Didáctica y Organización Educativa en la Universidad de Sevilla. Miembro del Grupo de Investigación Didáctica: Análisis Tecnológico y Cualitativo de los Procesos de Enseñanza-Aprendizaje (Plan Andaluz de Investigación: HUM-390). Director de la Cátedra Paulo Freire en la Universidad de Sevilla. Coordinador del Máster en Formación y Orientación para el Trabajo. Más información del CV en: http://investigacion.us.es/sisius/sis showpub.php?idpers $=1950$

E-mail: jamorales@us.es 


\section{DIRECCIÓN DE LOS AUTORES}

Universidad de Sevilla

Facultad de Ciencias de la Educación

Departamento de Didáctica y Organización Educativa

C/ Pirotecnia, $\mathrm{s} / \mathrm{n}$

41013 Sevilla (España)

Fecha de recepción del artículo: 01/10/2016

Fecha de aceptación del artículo: 02/11/2016

\section{Como citar este artículo:}

Cabero Almenara, J., Llorente Cejudo, M. C., y Morales Lozano, J. A. (2018). Evaluación del desempeño docente en la formación virtual: ideas para la configuración de un modelo. RIED. Revista Iberoamericana de Educación a Distancia, 21(1), pp. 261-279. doi: http://dx.doi.org/10.5944/ried.21.1.17206 